\title{
Secukinumab: A Review in Psoriatic Arthritis
}

\author{
Hannah A. Blair ${ }^{1}$ \\ Accepted: 2 February 2021 / Published online: 4 March 2021 \\ (c) Springer Nature 2021, corrected publication 2021
}

\begin{abstract}
Secukinumab $\left(\right.$ Cosenty $\left.^{\circledR}{ }^{\circledR}\right)$ is a fully human monoclonal antibody that selectively targets interleukin (IL)-17A, a proinflammatory cytokine involved in the pathogenesis of psoriatic arthritis (PsA). Administered subcutaneously, the first-in-class anti-IL-17 agent is approved in numerous countries worldwide for the treatment of adults with active PsA. In the phase III FUTURE trials, secukinumab 150 or $300 \mathrm{mg}$ improved the clinical signs and symptoms of PsA versus placebo in patients with active disease despite previous treatment with NSAIDs, biological disease-modifying anti-rheumatic drugs (bDMARDs) and/or tumour necrosis factor inhibitors (TNFi). The benefits of secukinumab were seen regardless of whether or not patients had received previous TNFi therapy, and were maintained during longer term (up to 5 years) treatment. In FUTURE 1 and 5 , secukinumab inhibited structural joint damage and was associated with sustained low rates of radiographic progression through 1-3 years of treatment. Treatment with secukinumab improved physical function and health-related quality of life (HR-QOL) and was generally well tolerated, both in the short- and longer-term. In the head-to-head EXCEED trial, secukinumab did not quite attain statistical significance for superiority versus adalimumab in the joint domain. In conclusion, secukinumab is effective across all key PsA domains and is generally well tolerated, and thus represents a useful treatment alternative to TNFi and other bDMARDs in adult patients with active PsA.
\end{abstract}

\section{Introduction}

Psoriatic arthritis (PsA) is a chronic inflammatory musculoskeletal condition characterized by a wide range of symptoms including arthritis, spondylitis, dactylitis, enthesitis, psoriasis and nail disease $[1,2]$. PsA is also associated with several comorbidities [1-3], and can significantly worsen healthrelated quality of life (HR-QOL) [1]. Traditional pharmacological treatment options for PsA include NSAIDs and conventional synthetic disease-modifying anti-rheumatic drugs

\section{Digital Features for this Adis Drug Evaluation can be found at} https://doi.org/10.6084/m9.figshare.13681804.

The manuscript was reviewed by: $\boldsymbol{J}$. Gratacos, Rheumatology Department, Universitat Autonoma de Barcelona, Parc Tauli Hospital Universitari, Barcelona, Spain; G. Han, Icahn School of Medicine at Mount Sinai, New York, NY, USA; D. Wendling, Service de Rhumatologie, Centre Hospitalier Universitaire de Besancon, Besancon, France.

Hannah A. Blair

demail@springer.com

1 Springer Nature, Mairangi Bay, Private Bag 65901, Auckland 0754, New Zealand
(csDMARDs) such as methotrexate [1, 3]. Over recent decades, the development of biological DMARDs (bDMARDs) has considerably changed the PsA treatment landscape [1]. The first bDMARDs to be approved were the tumour necrosis factor inhibitors (TNFi), based on the finding that TNF- $\alpha$ is a key mediator of acute inflammation in PsA [4]. In addition to TNF- $\alpha$, other proinflammatory cytokines, such as interleukin (IL)-12, IL-17 and IL-23, have been shown to play a key role in the pathogenesis of PsA $[1,4,5]$. IL-17A (a member of the IL-17 family) is up-regulated in psoriatic lesional skin and in the synovial fluid of PsA patients $[1,4,5]$, providing a rationale for the development of targeted anti-IL-17 therapies [5].

Secukinumab $\left(\right.$ Cosentyx $\left.^{\circledR}\right)$ is a recombinant, high affinity, fully human monoclonal antibody targeted against IL-17A. It is approved in numerous countries, including the USA [6] and those of the EU [7], for the treatment of adult patients with active PsA. The pharmacological properties of secukinumab have been reviewed in detail previously [8] and are summarized in Table 1. This review focuses on the clinical use of secukinumab in patients with PsA. Secukinumab is also approved for the treatment of plaque psoriasis [9], ankylosing spondylitis (AS) [10,11] and non-radiographic axial spondyloarthritis (nr-axSpA); however, discussion of the use of secukinumab in these indications is beyond the scope of this review. 


\section{Secukinumab: clinical considerations in psoriatic} arthritis

First-in-class fully human monoclonal antibody targeted against IL-17A

Improves clinical signs and symptoms, physical function and HR-QOL, with benefits sustained over the longer term (up to 5 years)

Associated with low rates of radiographic progression

Effective in both TNFi-naïve and -experienced patients and regardless of concomitant methotrexate use

Generally well tolerated

\section{Therapeutic Efficacy of Secukinumab}

The efficacy of secukinumab for the treatment of PsA was evaluated in several randomized, double-blind, placebocontrolled, multicentre, phase III trials: FUTURE 1-5 [12-16] (Sect. 2.1.1), MAXIMISE [17] (Sect. 2.1.2) and ULTIMATE [18] (Sect. 2.1.3). These data are supported by a randomized, double-blind, active-controlled, multicentre, phase IIIb trial comparing secukinumab with adalimumab (EXCEED) [19] (Sect. 2.2). The efficacy of secukinumab in the real-world setting is also briefly discussed (Sect. 2.3).

\subsection{Versus Placebo}

\subsubsection{FUTURE Trials}

The FUTURE trials enrolled patients aged $\geq 18$ years with a diagnosis of PsA that met the Classification of Psoriatic Arthritis criteria [12-16]. All patients had active disease,

\section{Table 1 Overview of key pharmacological properties of secukinumab [6-8]}

\section{Pharmacodynamic properties}

Recombinant, high affinity, fully human IgG1/א monoclonal antibody; selectively binds to and neutralizes IL-17A and inhibits its interaction with the IL-17 receptor; inhibits the release of proinflammatory cytokines, chemokines and mediators of tissue damage

Initial $\uparrow$ in serum levels of total IL-17A (free + SEC-bound IL-17A), then slow $\downarrow$ due to reduced clearance of SEC-bound cytokine

Clinically relevant levels of SEC reach the skin, leading to $\downarrow$ local inflammatory markers and $\downarrow$ erythema, induration and desquamation

$\downarrow$ Psoriatic epidermal abnormalities (i.e. epidermal thickening, parakeratosis, acanthosis) with parallel $\downarrow$ in epidermal neutrophil counts in plaques; $\downarrow$ pathological infiltration

of psoriasis lesions by $\mathrm{T}$ cells, macrophages and inflammatory dendritic cell subsets

$\downarrow$ Synovial inflammation and no progression of catabolic and anabolic bone changes in joints of pts with PsA [66]

$\downarrow$ Levels of C-reactive protein (inflammatory biomarker) in pts with PP, PsA and AS

$\downarrow$ Serum levels of $\beta$-defensin 2 (proposed surrogate marker of disease activity) in pts with PsA

Pts receiving SEC should not be administered live vaccines; non-live vaccines may be given during SEC treatment; SEC did not impair immune response to a meningococcal polysaccharide conjugate vaccine and an inactivated influenza vaccine

Pharmacokinetic properties

Pharmacokinetics of SEC in pts with PsA are similar to those in pts with PP or other autoimmune diseases

Dose-proportional exposure over dose range of $25-300 \mathrm{mg}$; bioavailability $85 \%$; $\mathrm{C}_{\max }$ reached $\approx 6$ days following a single dose; mean $\mathrm{C}_{\text {max }}$ at steady state after loading and maintenance doses estimated to be 31 and $62 \mu \mathrm{g} / \mathrm{mL}$ with 150 and $300 \mathrm{mg}$ doses

Administration via Sensoready ${ }^{\circledR}$ pen resulted in mean trough concentrations at weeks 4 and 12 that were $23-30 \%$ higher than after administration of reconstituted lyophilized powder and $23-26 \%$ higher than after administration via prefilled syringe

Low total Vd; estimated Vd 3.66 and 2.45 L for the central and peripheral compartments

Majority of elimination occurs through intracellular catabolism (following endocytosis); systemic clearance $\approx 0.19$ L/day; average terminal elimination half-life 25 days

Special populations $^{\mathrm{b}} \uparrow$ SEC clearance and Vd with $\uparrow$ bodyweight

Hepatic impairment or abnormal kidney function is not expected to influence SEC elimination or clearance

No dosage adjustment required in pts aged $\geq 65$ years

No clinically relevant differences in SEC pharmacokinetics based on age, gender or race (after adjusting for bodyweight)

Drug interactions $^{\mathrm{b}} \quad$ No specific drug interaction studies have been performed

No interaction when SEC is coadministered with methotrexate \pm oral glucocorticoids

Formation of some CYP450 enzymes can be altered by $\uparrow$ levels of certain cytokines during chronic inflammation; SEC could potentially affect CYP450 levels; consider monitoring and dosage adjustment when initiating or discontinuing SEC in pts receiving concomitant CYP450 substrates (particularly those with a narrow therapeutic index)

$\downarrow$ decrease(d), $\uparrow$ increase(d), $A S$ ankylosing spondylitis, $C_{\max }$ maximum plasma concentration, $I g$ immunoglobulin, $I L$ interleukin, $P P$ plaque psoriasis, $P S A$ psoriatic arthritis, pts patients, $S E C$ secukinumab, $V d$ volume of distribution

${ }^{a}$ All pharmacokinetic parameters are for subcutaneous SEC in pts with PsA unless otherwise stated

${ }^{\mathrm{b}}$ Consult local prescribing information for detailed recommendations 
defined as $\geq 3$ tender joints and $\geq 3$ swollen joints, despite previous treatment with NSAIDs, DMARDs and/or TNFi. Patients who had previously received TNFi could enrol if they had had an inadequate response or had discontinued treatment due to safety or tolerability issues. Stable dosages of concomitant corticosteroids and methotrexate were allowed. Across all trials, the mean age of patients was 47-50 years, $65-76 \%$ of patients were TNFi-naïve and $47-61 \%$ were receiving concomitant methotrexate [12-16].

Patients in each trial were randomized to one of two [12, $14,15]$ or three $[13,16]$ secukinumab groups or a placebo group; randomization was stratified by previous use of TNFi [12-16]. In FUTURE 1, secukinumab recipients received intravenous loading doses of secukinumab $10 \mathrm{mg} / \mathrm{kg}$ at weeks 0,2 and 4, followed by subcutaneous secukinumab 75 or 150 mg every 4 weeks [12]. In FUTURE 2, secukinumab recipients received subcutaneous secukinumab 75 , 150 or $300 \mathrm{mg}$ at weeks $0,1,2,3$ and 4 , then every 4 weeks [13]. In FUTURE 3, subcutaneous secukinumab 150 or $300 \mathrm{mg}$ was self-administered via autoinjector at weeks 0 , 1, 2, 3 and 4, then every 4 weeks [14]. In FUTURE 4, subcutaneous secukinumab was administered with a loading regimen (i.e. $150 \mathrm{mg}$ at weeks $0,1,2$ and 3 , then every 4 weeks) or without a loading regimen (i.e. $150 \mathrm{mg}$ every 4 weeks) [15]. In FUTURE 5, subcutaneous secukinumab was self-administered via prefilled syringe with a loading regimen (i.e. 150 or $300 \mathrm{mg}$ at weeks $0,1,2$ and 3, then every 4 weeks) or without a loading regimen (i.e. $150 \mathrm{mg}$ every 4 weeks) [16]. Discussion in this section focuses on the approved doses of 150 and $300 \mathrm{mg}$ (Sect. 4), with data for the $75 \mathrm{mg}$ dose presented in Table 2 for completeness.

At week 16 in each trial, patients were classified as responders (i.e. improvement of $\geq 20 \%$ from baseline in the number of tender and swollen joints) or non-responders [12-16]. Patients in the placebo groups were then reassigned to receive secukinumab 75 or $150 \mathrm{mg}$ in FUTURE 1 [12], 150 or $300 \mathrm{mg}$ in FUTURE 2, 3 and 5 [13, 14, 16], and $150 \mathrm{mg}$ in FUTURE 4 [15] every 4 weeks from week 16 (non-responders) or week 24 (responders).

The primary endpoint was the proportion of patients achieving $\geq 20 \%$ improvement in the American College of Rheumatology response criteria (ACR20) at week 16 in FUTURE 4 and $5[15,16]$ and at week 24 in FUTURE 1, 2 and 3 [12-14].

2.1.1.1 Clinical Response Secukinumab improved the signs and symptoms of PsA, with ACR20 response rates at week 16 $[15,16]$ or week $24[12-14]$ being significantly higher with both doses of secukinumab (i.e. 150 and $300 \mathrm{mg}$ ) than with placebo (Table 2). In prespecified exploratory subgroup analyses, the benefits of secukinumab (although not always significant vs placebo) were seen regardless of whether patients had received previous TNFi or not [12-16]. For example,
ACR20 response rates at week $16[15,16]$ or week 24 [12-14] were significantly $(p<0.05)$ higher with secukinumab than with placebo among TNFi-naïve patients in all trials [12-16]. Among TNFi-experienced patients, ACR20 response rates were numerically higher with secukinumab than with placebo in all trials $[12-16]$ and significantly $(p<0.05)$ higher than with placebo in FUTURE 1,3 and $5[12,14,16]$. In prespecified (FUTURE 3) [14] or post hoc (FUTURE 1 and 2) [12, 13] analyses, ACR 20 response rates at week 24 were significantly higher with secukinumab than with placebo regardless of concomitant methotrexate use.

With regard to secondary endpoints, secukinumab was associated with improvements in several other clinical domains of PsA at week $16[15,16]$ or 24 [12-14]. The proportions of patients achieving $\geq 50 \%$ improvement in the ACR response criteria (ACR50) and $\geq 75$ and $\geq 90 \%$ improvement in Psoriasis Area-and-Severity Index (PASI) scores (PASI75 and PASI90) were significantly higher with secukinumab than with placebo in all trials, with the exception of ACR50 with secukinumab $150 \mathrm{mg}$ in FUTURE 2 and PASI90 with secukinumab $150 \mathrm{mg}$ in FUTURE 3 (Table 2). Changes from baseline in 28-joint Disease Activity Score using C-reactive protein levels (DAS28-CRP) were significantly greater with secukinumab than with placebo in all trials (Table 2). Secukinumab provided resolution of dactylitis and enthesitis among patients who had these disease characteristics at baseline, although rates of resolution were not always significant versus placebo (Table 2) [12-16].

The clinical responses reported with secukinumab at week 16 or 24 were maintained through 52 weeks [12-15, 20] and 104 weeks [15, 21-23] of treatment. For example, ACR20 response rates (with multiple imputations and as observed data, where reported) ranged from 47 to $73 \%$ at week $52[12-15,20]$ and from 64 to $73 \%$ at week 104 [15, 21-23]. Over the longer term, secukinumab provided sustained improvements in ACR20 response rates and other key efficacy endpoints for up to 5 years in FUTURE $1[24,25]$ and FUTURE 2 [26, 27]. For example, ACR20 response rates at 5 years remained high $(70-74 \%)[24,27]$.

2.1.1.2 Radiological Response Secukinumab was associated with low rates of radiographic structural progression in patients with PsA $[12,16]$. In FUTURE 1 and 5, the change from baseline in van der Heijde-modified total Sharp score (vdH-mTSS; higher scores indicate more damage) at week 24 was significantly $(p<0.05)$ lower among secukinumab than placebo recipients $[12,16]$. The inhibition of radiographic progression achieved with secukinumab at week 24 was maintained through 52 weeks [20, 28], 104 weeks [21, 23] and 156 weeks [25] of treatment. Moreover, switching to secukinumab inhibited further radiographic progression in patients initially randomized to placebo [20]. 


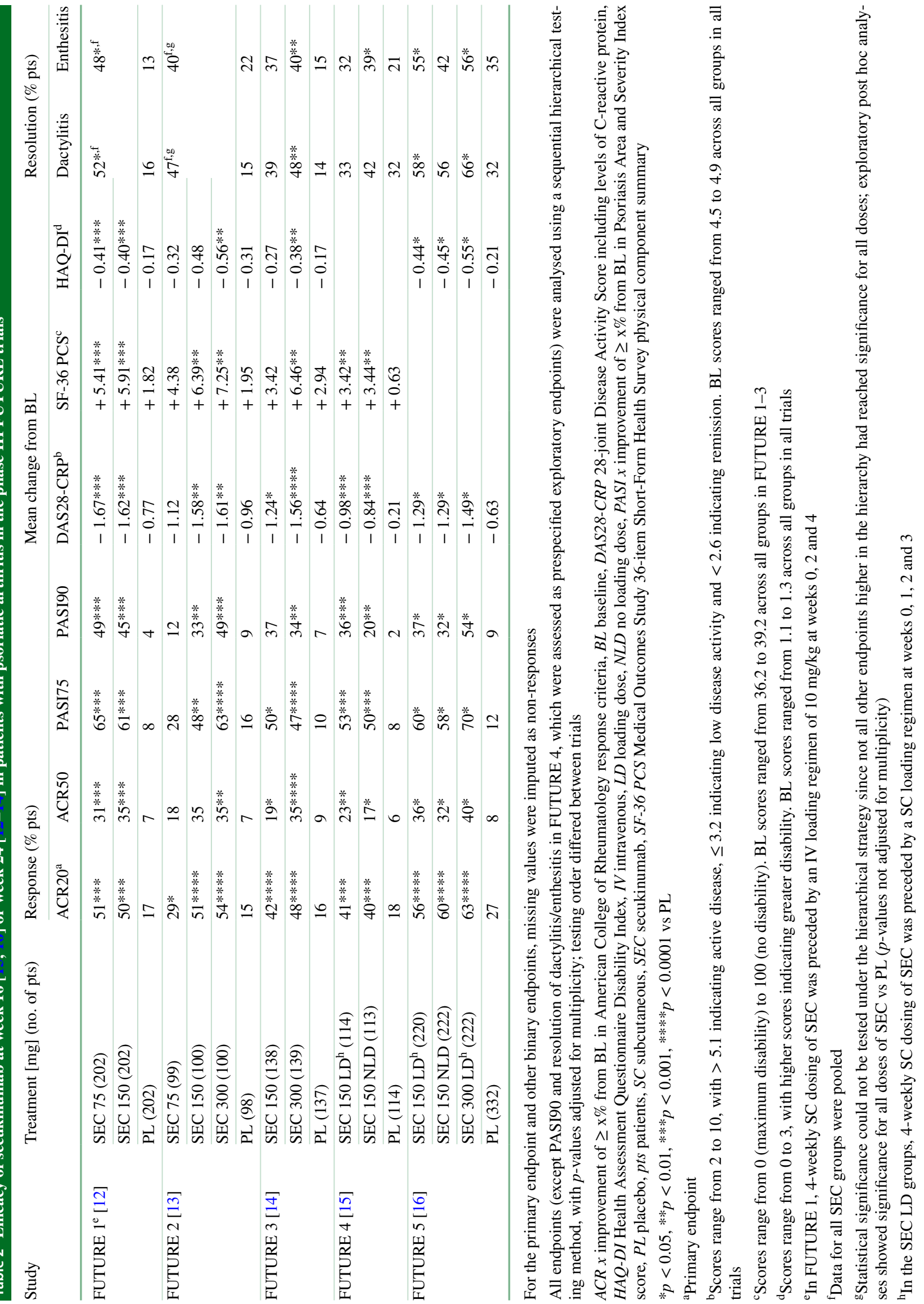


In subgroup analyses, low rates of radiographic progression were observed with secukinumab regardless of prior exposure to TNFi $[20,28]$ and whether patients were receiving concomitant methotrexate [28]. At week 52, the majority (85-92\%) of patients receiving secukinumab had no radiographic progression, defined as $\leq 0.5$ change from baseline in vdH-mTSS $[20,28]$.

2.1.1.3 Patient-Reported Outcomes Secukinumab improved physical function in patients with PsA, as indicated by significantly greater changes from baseline in Health Assessment Questionnaire Disability Index (HAQ-DI) scores at week $16[15,16]$ or $24[12-14]$ with secukinumab $150 \mathrm{mg}$ versus placebo in FUTURE 1 and $5[12,16]$ and with secukinumab $300 \mathrm{mg}$ versus placebo in FUTURE 2, 3 and 5 $[13,14,16]$ (Table 2). In FUTURE 4, the HAQ-DI response rate at week 16 was $34 \%$ in the secukinumab load group, $43 \%$ in the secukinumab no-load group $(p<0.01$ vs placebo) and $29 \%$ in the placebo group [15]. Improvements in physical function seen with secukinumab at week 16 or 24 were maintained for up to 5 years in FUTURE 1 [23-25, 29] and FUTURE 2 [22, 26, 27].

Secukinumab improved HR-QOL, as assessed by the Medical Outcomes Study 36-item Short-Form Health Survey (SF-36) [12-16] and the Functional Assessment of Chronic Illness Therapy (FACIT) measurement system [14, 15, 22, 29]. At week 16 [15] or 24 [12-14], secukinumab was associated with significantly greater improvements from baseline than placebo in SF-36 physical component summary scores (Table 2) [12-15] as well as FACIT-Fatigue scores [14, 15, 22, 29]. At week 24 in FUTURE 1 and 2, secukinumab was also associated with improvements from baseline in visual analogue scale (VAS) pain scores, PsA QOL, Work Productivity and Activity Impairment-General Health, Dermatology Life Quality Index and EuroQol 5-Dimension Health Status Questionnaire scores compared with placebo, with continued or additional improvements in these scores at week 52 [29] and week 104 [22, 30]. Improvements in HRQOL were sustained over the longer term (up to 5 years) in FUTURE 1 [23-25] and FUTURE 2 [27].

In FUTURE 3, > 99\% of patients reported successful self-administration of secukinumab using the autoinjector at week 1 [14]. Self-Injection Assessment Questionnaire scores for feelings about injections, self-confidence and satisfaction with self-injection were high. At week $2, \geq 88 \%$ of patients were satisfied or very satisfied with the use of the autoinjector and found the device easy or very easy to use [14].

2.1.1.4 Disease Activity Endpoints Minimal disease activity (MDA), a composite measure including multiple PsA domains, was assessed in FUTURE 2 [31] and as a prespecified exploratory endpoint in FUTURE 4 and 5 [15, 16]. Significantly $(p<0.05)$ more secukinumab than pla- cebo recipients achieved an MDA response (i.e. fulfilling $\geq 5 / 7$ criteria) at week $16[15,16,31]$, with response rates sustained through 52 weeks in FUTURE 4 [15] and 5 years in FUTURE 2 [27]. In FUTURE 2, 40-49\% of MDA responders met the more stringent criteria for very low disease activity (VLDA; fulfilling 7/7 criteria) at 2 years [31].

Post hoc analyses assessed the ability of secukinumab to achieve remission or low disease activity (LDA) using Disease Activity Index for Psoriatic Arthritis (DAPSA) and Psoriatic Arthritis Disease Activity Score (PASDAS) scores $[16,32,33]$. In FUTURE 2 and 5, more secukinumab than placebo recipients achieved DAPSA remission (score of $\leq 4$ ) or LDA (score of $>4$ to $\leq 14$ ) at week 16 [16, 33], with responses maintained through 2 years [33]. In FUTURE 2, more patients achieved DAPSA remission (score of $\leq 4$ ) compared with VLDA, and DAPSA remission + LDA (score of $\leq 14$ ) compared with MDA [33]. More secukinumab than placebo recipients achieved PASDAS remission (score of $\leq 1.9$ ) or LDA (score of $>1.9$ to $<3.2$ ) at week 16 , with responses sustained through 2 years [32].

\subsubsection{MAXIMISE Trial}

The MAXIMISE trial enrolled patients aged $\geq 18$ years with PsA and active spinal disease with a spinal pain VAS score of $\geq 40 / 100$ and a Bath Ankylosing Spondylitis Disease Activity Index score of $\geq 4$ despite the use of at least two NSAIDs [17]. Patients were randomized to receive subcutaneous secukinumab $150 \mathrm{mg}(n=165)$, secukinumab $300 \mathrm{mg}$ $(n=167)$ or placebo $(n=166)$ weekly for 4 weeks and then every 4 weeks. At week 12, patients in the placebo group were re-randomized to secukinumab 150 or $300 \mathrm{mg}$. The primary endpoint was the proportion of patients achieving $\geq 20 \%$ improvement in the Assessment of Spondyloarthritis International Society response criteria (ASAS20) at week 12 [17].

Secukinumab improved axial manifestations in patients with PsA [17]. An ASAS20 response at week 12 was achieved by 66 and $63 \%$ of secukinumab 150 and $300 \mathrm{mg}$ recipients, compared with $31 \%$ of placebo recipients; the odds ratios (ORs) for secukinumab 150 and $300 \mathrm{mg}$ versus placebo were 4.4 (95\% CI 2.7-7.0) and 3.8 (95\% CI 2.4-6.1; both $p<0.0001)$. Similar ASAS20 response rates were seen in the subgroup of patients with positive MRI for spine and/ or sacroiliac joints at baseline ( 66 and $70 \%$ vs $27 \%$ ). ASAS20 response rates continued to increase through 52 weeks of treatment. The observed ASAS20 response rates at week 52 were $80 \%$ in the secukinumab $150 \mathrm{mg}$ group, $81 \%$ in the secukinumab $300 \mathrm{mg}$ group, $80 \%$ in patients who switched from placebo to secukinumab $150 \mathrm{mg}$ at week 12 and $75 \%$ in patients who switched from placebo to secukinumab $300 \mathrm{mg}$ at week 12. Secukinumab also reduced inflammation in the spine and sacroiliac joints (exploratory endpoint). Both doses of secukinumab significantly improved Berlin MRI 
scores for the entire spine $(p<0.05)$ and the sacroiliac joint $(p<0.01)$ versus placebo at week 12 , providing evidence of response in the objective signs of inflammation; these responses were sustained through week 52 [17].

\subsubsection{ULTIMATE Trial}

The ULTIMATE trial enrolled patients with PsA who had joint synovitis on power doppler ultrasonography and at least one clinical enthesitis site [18]. All patients were biologicalnaïve with active PsA and had an inadequate response to csDMARDs. They were randomized to receive subcutaneous secukinumab 150 or $300 \mathrm{mg}(n=83)$ or placebo $(n=83)$ weekly for 4 weeks and then every 4 weeks. The primary endpoint was the difference between secukinumab and placebo in mean change from baseline to week 12 in the global OMERACT-EULAR synovitis score (GLOESS) [18].

Secukinumab significantly reduced joint synovitis in patients with PsA [18]. The least squares mean change in GLOESS at week 12 was significantly $(p=0.004)$ greater with secukinumab than with placebo $(-9.0$ vs -5.8$)$; improvement was seen as early as week 1 . Secukinumab was also associated with significantly $(p<0.0001)$ higher ACR20 (68 vs 34\%) and ACR50 (46 vs 9\%) response rates than placebo. The least squares mean change from baseline in Spondyloarthritis Research Consortium of Canada enthesitis index was -2.35 with secukinumab versus -1.65 with placebo $(p=0.02)$ [18].

\subsection{Versus Adalimumab}

The head-to-head EXCEED trial enrolled patients aged $\geq 18$ years with active PsA, defined as $\geq 3$ tender joints and $\geq 3$ swollen joints [19]. They also had active plaque psoriasis with $\geq 1$ plaque of $\geq 2 \mathrm{~cm}$ diameter or nail changes consistent with psoriasis or documented history of plaque psoriasis. All patients were biological-naïve, had an inadequate response or were intolerant to csDMARDs (including, but not limited to, methotrexate) and had an inadequate response to NSAIDs for $\geq 4$ weeks prior to randomization. Stable dosages of concomitant NSAIDs and corticosteroids were permitted. Following a washout period of 4 weeks for all csDMARDs or 8 weeks for leflunomide, patients were randomized to receive subcutaneous secukinumab $300 \mathrm{mg}$ via prefilled syringe at weeks $0,1,2,3$ and 4 , then every 4 weeks until week $48(n=426)$ or subcutaneous adalimumab $40 \mathrm{mg}$ every 2 weeks until week $50(n=427)$ [19].

Secukinumab was not more effective than adalimumab in the joint domain in patients with PsA [19]. The ACR20 response rate at week 52 (primary endpoint) was $67 \%$ with secukinumab and $62 \%$ with adalimumab (Table 3 ). In a prespecified sensitivity analysis using non-responder imputation, respective ACR20 response rates were 67 and 59\% (OR $1.38 ; 95 \%$ CI $1.04-1.83 ; p=0.0239)$. Because the superiority of secukinumab versus adalimumab for the primary endpoint was not met, statistical significance could not be formally tested for the key secondary endpoints in the hierarchy: PASI90, ACR50, change from baseline in HAQ-DI and resolution of enthesitis (Table 3). Of note, the PASI90 response rate was numerically higher with secukinumab than with adalimumab (Table 3). Overall, 14\% of secukinumab recipients and $24 \%$ of adalimumab recipients discontinued treatment by week 52 [19].

\subsection{Real-World Setting}

Real-world experience has confirmed the efficacy of secukinumab for the treatment of PsA. In several real-world studies $(n \geq 100)$ conducted mainly in Europe and the USA, secukinumab improved clinical outcomes (e.g. PsA symptoms,

Table 3 Efficacy of secukinumab versus adalimumab at week 52 in patients with psoriatic arthritis in the phase III EXCEED trial [19]

\begin{tabular}{llll} 
Endpoint (full analysis set) & SEC 300 $\mathrm{mg}(n=426)$ & ADA $40 \mathrm{mg}(n=427)$ & OR (95\% CI) \\
\hline ACR20 (\% pts) & 67 & 62 & $1.30(0.98-1.72)$ \\
\hline PASI90 (\% pts) & $65^{* *}$ & 43 & $2.49(1.67-3.71)$ \\
\hline ACR50 (\% pts) & 49 & 45 & $1.18(0.90-1.55)$ \\
\hline Change from BL in HAQ-DI & -0.58 & -0.56 & $-0.02^{\mathrm{b}}(-0.10$ to 0.05$)$ \\
\hline Resolution of enthesitis $(\%$ pts) & 61 & 54 & $1.30(0.91-1.87)$ \\
ACR50 + PASI100 (\% pts) & $31^{*}$ & 19 & $1.85(1.17-2.92)$ \\
\hline
\end{tabular}

$A C R x$ improvement of $\geq \mathrm{x} \%$ from $\mathrm{BL}$ in American College of Rheumatology response criteria, $A D A$ adalimumab, $B L$ baseline, $H A Q-D I$ Health Assessment Questionnaire Disability Index, OR odds ratio, PASI $x$ improvement of $\geq \mathrm{x} \%$ from BL in Psoriasis Area and Severity Index score, pts patients, $S E C$ secukinumab

$* p=0.0087, * * p<0.0001$ vs ADA (unadjusted)

${ }^{\text {a Primary endpoint }}$

${ }^{\mathrm{b}}$ Between-treatment difference

${ }^{c}$ Based on Leeds Enthesitis Index 
disease activity, remission rates) [34-41] and PROs (e.g. pain, QOL) [34-37] in patients with PsA. Secukinumab was also associated with high levels of patient $(\geq 96 \%)[40]$ and physician (88\%) [42] satisfaction, and high (56-86\%) rates of adherence, retention and persistence [38, 43, 44].

In large observational studies comparing adherence, retention and persistence rates with various bDMARDs in patients with PsA, results were mixed. In a retrospective cohort study using US pharmacy claims data $(n=1558)$, the 12-month discontinuation rate was lowest with secukinumab $(37 \%)$, followed by adalimumab (43\%), golimumab (45\%), etanercept (48\%) and certolizumab pegol (52\%) [45]. Mean persistence ranged from 241 days with certolizumab pegol to 283 days with secukinumab. The proportion of patients who remained adherent to treatment was highest with secukinumab (46\%), followed by adalimumab (44\%), golimumab (39\%), etanercept $(37 \%)$ and certolizumab pegol $(18 \%)$ [45]. In a French cohort study $(n=406)$, the persistence of secukinumab at 2 years was higher than that of ustekinumab [46]. In an observational study using data from a Nordic collaboration $(n=6062), 1$-year treatment retention rates with secukinumab (63-72\%) were comparable to those with adalimumab (67-73\%), certolizumab pegol (49-66\%), etanercept (62-73\%), golimumab (56-69\%) and infliximab (57-65\%) [47].

\section{Tolerability of Secukinumab}

Subcutaneous secukinumab 150 or $300 \mathrm{mg}$ was generally well tolerated in clinical trials, including in patients with PsA, plaque psoriasis, AS and other autoimmune conditions [7]. The tolerability profile was consistent across all indications. The most common adverse events (AEs) with secukinumab in clinical trials and post-marketing reports were upper respiratory tract infections (URTIs), most commonly nasopharyngitis and rhinitis [7].

During the 16- or 24-week placebo-controlled periods of FUTURE 1-3 and 5, AEs occurred in 55-65\% of secukinumab recipients and 56-62\% of placebo recipients, with serious AEs reported in $1-5 \%$ of secukinumab and $2-7 \%$ of placebo recipients, and discontinuations due to AEs reported in $0-4 \%$ of secukinumab and $2-4 \%$ of placebo recipients [12-14, 16]. During this period, the most common (incidence $\geq 5 \%$ ) AEs with secukinumab were URTI, nasopharyngitis and headache [12-14, 16].

In the head-to-head EXCEED trial, the safety profiles of secukinumab and adalimumab were consistent with previous reports [19]. During the 52-week treatment period, AEs were reported in $77 \%$ of secukinumab recipients and $79 \%$ of adalimumab recipients. The most common (incidence $\geq 10 \%$ ) AEs were nasopharyngitis (19\% with secukinumab vs $19 \%$ with adalimumab) and URTI (10 vs 11\%). Injection-site reactions occurred in $4 \%$ of secukinumab recipients and $11 \%$ of adalimumab recipients. Four percent of patients in the secukinumab group and $7 \%$ of patients in the adalimumab group discontinued treatment due to AEs [19].

Over the entire safety periods of FUTURE 1-5 (52 weeks in FUTURE 3 and 5 [14, 20], 104 weeks in FUTURE 4 [15], and 260 weeks in FUTURE 1 and 2 [24, 27]), the long-term tolerability profile of secukinumab was generally consistent with that reported previously, with no new or unexpected safety signals. The exposure-adjusted incidence rate (EAIR) of any AE during 5 years of secukinumab treatment was 126.7 per 100 patient-years (PY) in FUTURE 1 [24] and 140.5 per $100 \mathrm{PY}$ in FUTURE 2 [27]. In a pooled analysis of FUTURE 1-3 ( $n=1380$; total exposure 3866.9 PY), the EAIR of any AE with secukinumab across the entire safety period was 147.0 per 100 PY [48]. Post-marketing safety surveillance data based on cumulative secukinumab exposure of $\approx 285,811 \mathrm{PY}$ across three indications (PsA, plaque psoriasis and AS) confirmed that secukinumab was generally well tolerated over the longer-term [49].

\subsection{Adverse Events of Special Interest}

Treatment with secukinumab may increase the risk of infections $[6,7]$, with the incidence of some types of infections appearing to be dose-dependent [6]. In placebo-controlled trials in patients with PsA, plaque psoriasis, AS or nr-axSpA, higher rates of common infections such as URTI, nasopharyngitis and mucocutaneous Candida infections were observed with secukinumab relative to placebo $[6,7]$. However, these infections were of mild to moderate severity and did not require discontinuation of secukinumab [7]. In a pooled analysis of FUTURE 1-5 ( $n=2678$; total exposure 5984.6 PY), serious infections and Candida infections occurred at EAIRs of 1.8 and 1.5 per $100 \mathrm{PY}$ of exposure to secukinumab during the entire safety period [49]. Caution is advised when considering the use of secukinumab in patients with chronic infections or a history of recurrent infection [6,7]. If signs or symptoms of an infection occur, patients should seek medical advice. Patients who develop a serious infection while receiving secukinumab should be closely monitored and secukinumab should be discontinued until the infection resolves $[6,7]$.

No cases of active tuberculosis (TB) or latent TB infection reactivation were reported in the secukinumab clinical trial programme [50]. However, patients should be evaluated for TB infection prior to initiating secukinumab, and should be closely monitored for signs and symptoms of active TB during and after treatment [6]. Secukinumab should not be given to patients with active TB, and anti-TB therapy should be considered prior to initiation of secukinumab in patients with latent TB $[6,7]$. 
There have been reports of new-onset or exacerbations of inflammatory bowel disease (IBD), including some serious cases, in patients receiving secukinumab $[6,7]$. In the pooled analysis of FUTURE 1-5, the EAIRs of IBD, Crohn's disease and ulcerative colitis with secukinumab during the entire safety period were $0.03,0.1$ and 0.1 per $100 \mathrm{PY}$, respectively [49]. Data on a year-on-year basis showed no increase in the EAIR of IBD with secukinumab treatment over time [49]. Caution is advised when prescribing secukinumab to patients with IBD, and patients receiving secukinumab should be closely monitored for signs and symptoms of IBD [6, 7].

Hypersensitivity reactions including anaphylaxis and urticaria have occurred in patients receiving secukinumab [6, 7]. Serious allergic reactions to secukinumab should be treated with appropriate therapy, and the drug should be discontinued immediately $[6,7]$.

In the pooled analysis of FUTURE 1-5, the EAIRs for a number of other AEs of special interest were low and stable during the entire safety period, namely malignancy (1.0 per $100 \mathrm{PY}$ ), major adverse cardiovascular events (MACE; 0.4 per $100 \mathrm{PY}$ ) and uveitis (0.1 per $100 \mathrm{PY}$ ) [49]. Similar results were seen in post-marketing surveillance. Across five consecutive periodic safety update reporting periods between December 2014 and December 2018, the rates of serious infections, malignancy, total IBD and MACE were 1.4, 0.3, 0.2 and 0.2 per $100 \mathrm{PY}$, respectively [49].

Like all therapeutic proteins, secukinumab has the potential for immunogenicity [6]. Over 52 weeks of treatment in FUTURE 1-3, five (0.4\%) secukinumab recipients developed anti-drug antibodies (ADAs) [51]. In four of these cases, the antibodies were non-neutralizing. The emergence of ADAs was not associated with immunogenicity-related AEs, loss of clinical response or deviations in the expected pharmacokinetics of secukinumab [51].

\section{Dosage and Administration of Secukinumab}

Subcutaneous secukinumab is approved in the USA [6] and the EU [7] for the treatment of adult patients with active PsA (when the response to previous DMARD therapy has been inadequate [7]), and may be administered with or without concomitant methotrexate $[6,7]$. Secukinumab is available as a lyophilized powder $(150 \mathrm{mg})$ in a vial for reconstitution, or as a $150 \mathrm{mg} / \mathrm{mL}$ solution for injection in a prefilled syringe or Sensoready ${ }^{\circledR}$ pen. The lyophilized powder for reconstitution is to be administered by healthcare providers only, while secukinumab in a prefilled syringe or pen may be self-injected after proper training in subcutaneous injection technique [6, 7]. Each injection should be administered at a different site (e.g. upper arm, thigh or abdomen) than the previous injection [6], avoiding areas of skin affected by psoriasis (if possible) [6, 7].

The recommended dose of secukinumab in patients with PsA and concomitant moderate to severe plaque psoriasis (or an inadequate response to TNFi [7]) is $300 \mathrm{mg}$ administered at weeks $0,1,2,3$ and 4, followed by 4-weekly (USA) or monthly (EU) maintenance dosing [6, 7]. For other patients with PsA, the recommended dose of secukinumab is $150 \mathrm{mg}$ administered at weeks $0,1,2,3$ and 4 (i.e. loading doses), followed by 4-weekly (USA) or monthly (EU) maintenance dosing [6, 7]; or alternatively, in the USA [6], $150 \mathrm{mg}$ administered every 4 weeks without loading doses. The dose of secukinumab can be increased to $300 \mathrm{mg}$ based on clinical response [7] or if active disease persists [6]. Consult local prescribing information for further detailed information regarding contraindications, warnings and precautions, drug interactions and use in special populations.

\section{Place of Secukinumab in the Management of Psoriatic Arthritis}

The main goals in the treatment of PsA are to control symptoms, prevent structural damage, optimize physical function and improve HR-QOL [52]. The choice of treatment is dependent on the clinical presentation of the disease. Consideration should be given to each musculoskeletal manifestation, as well as to non-musculoskeletal manifestations and comorbidities [52]. Treatment guidelines from the Group for Research and Assessment of Psoriasis and PsA (GRAPPA) [53] and EULAR [52] recommend a csDMARD (preferably methotrexate [52]) as first-line therapy for peripheral arthritis, followed by a bDMARD [i.e. TNFi, interkeukin-12/23 inhibitor (IL-12/23i) or interleukin-17 inhibitor (IL-17i)] if treatment targets are not achieved. In patients with predominantly axial disease, EULAR guidelines recommend the use of an IL-17i over a TNFi when there is relevant skin involvement [52]. Joint ACR and National Psoriasis Foundation (NPF) guidelines conditionally recommend a TNFi over an oral small molecule (i.e. csDMARD or apremilast) for treatment-naïve PsA, with IL-12/23i and IL-17i conditionally recommended after TNFi or in patients with contraindications to TNFi [54].

Secukinumab is a fully human monoclonal antibody that inhibits the proinflammatory effects of IL-17A (Table 1), and was the first IL-17 $\mathrm{i}$ to be approved for the treatment of active PsA (Sect. 4). Subcutaneous secukinumab 150 or $300 \mathrm{mg}$ was effective in the treatment of PsA in phase III clinical trials (Sect. 2). In the FUTURE trials, which included patients with different subtypes of PsA $[6,7]$, secukinumab improved clinical signs and symptoms compared with placebo, with these benefits maintained over the longer term 
(Sect. 2.1.1.1). The efficacy of secukinumab was seen across all key clinical domains of PsA, including ACR and PASI response rates, DAS28-CRP, and resolution of dactylitis and enthesitis. Of note, secukinumab was associated with clinically relevant improvements in some of the more stringent endpoints, such as ACR50, PASI90 (Sect. 2.1.1.1), MDA, VLDA and DAPSA remission (Sect. 2.1.1.4).

The clinical benefits of secukinumab were seen regardless of whether patients had or had not received previous TNFi and irrespective of concomitant methotrexate use (Sect. 2.1.1.1). The finding that secukinumab may be effective in both TNFi-naïve and -experienced patients is important, given that GRAPPA and EULAR treatment guidelines recommend switching to either another TNFi or to a different bDMARD class (i.e. IL-17i or IL-12/23i) if treatment targets are not met with the first TNFi [52, 53]. Current ACR/NPF guidelines conditionally recommend switching to a different TNFi after failure of the first TNFi [54].

PsA is a chronic and multifaceted disease which has a significant impact on HR-QOL in terms of physical dysfunction, pain, fatigue, work disability and emotional/social impairment [4]. In clinical trials, secukinumab was associated with clinically meaningful improvements in patientreported measures of physical function and HR-QOL (including pain, fatigue and work productivity) compared with placebo, with these improvements sustained over the longer term (Sect. 2.1.1.3).

Bone erosions are evident in approximately half of patients with PsA within 2 years of diagnosis, and many patients experience irreversible joint damage and disability [16]. Therefore, the prevention of structural damage is a fundamental goal in the management of PsA [52]. In FUTURE 1 and 5, secukinumab inhibited structural joint damage, with sustained low rates of radiographic progression seen over the longer term (Sect. 2.1.1.2). Notably, switching to secukinumab inhibited further radiographic progression in patients initially randomized to placebo (Sect. 2.1.1.2).

Axial disease is reported to affect $25-70 \%$ of patients with longstanding PsA [17]. Its burden of disease is underestimated, and it is distinct from axial spondyloarthritis [17]. The effect of secukinumab on axial disease was not assessed in the FUTURE trials, which was specifically noted as a potential limitation of FUTURE 1 and 2 [12, 13]. MAXIMISE was the first randomized controlled trial to evaluate the efficacy of a biological agent in the management of axial manifestations of PsA [17]. Final 52-week results demonstrated that secukinumab improved ASAS responses and reduced inflammatory MRI lesions in the spine and sacroiliac joints in PsA patients with axial involvement (Sect. 2.1.2). Further clinical trials with other bDMARDs would be of interest.
In the head-to-head EXCEED trial, secukinumab did not quite attain statistical significance for superiority versus the TNFi adalimumab in the joint domain (Sect. 2.2). Nevertheless, the trial provides new information regarding the comparative efficacy of two bDMARDs with differing mechanisms of action [55], and may help guide clinical decision-making regarding treatment choices for PsA after csDMARD failure or intolerance [19]. Additional well-designed clinical trials directly comparing secukinumab with other pharmacological agents in patients with PsA are needed. A phase III head-to-head trial comparing the efficacy of secukinumab and the IL-12/23i ustekinumab in patients with PsA and TNFi failure (AGAIN; NCT04632927) is currently underway. In addition, a number of systematic reviews and meta-analyses have indirectly compared the efficacy of bDMARDs (e.g. TNFi, ustekinumab, the IL-17i ixekizumab, secukinumab) and targeted synthetic DMARDs (e.g. apremilast) in patients with PsA [56-61]. However, the findings of these analyses require cautious interpretation due to the indirect nature of the comparisons and the heterogeneity of the studies and patient populations assessed.

Secukinumab was generally well tolerated in clinical trials (Sect. 3). The tolerability profile of secukinumab in patients with PsA was consistent with the profile of the drug used in other approved indications, including plaque psoriasis and AS. Secukinumab remained generally well tolerated over the longer term (up to 5 years), with no new or unexpected safety signals. Post-marketing safety surveillance data based on cumulative secukinumab exposure of $>285,000 \mathrm{PY}$ were consistent with data from clinical trials (Sect. 3).

Treatment retention is a composite measure of a drug's efficacy and tolerability [47], and low adherence rates and/or high discontinuation rates may indicate suboptimal treatment [45]. In real-world studies, rates of adherence, retention and persistence with secukinumab were better than or similar to those with other bDMARDs (Sect. 2.3). Additional studies would help to further elucidate the position of secukinumab in the real-world setting. The ongoing, longitudinal, observational SERENA study $(n=2932)$ will assess the long-term (up to 5 years) retention of secukinumab in routine clinical practice across three indications (plaque psoriasis, AS and PsA) [62].

The choice of treatment for PsA is individualized and should take into account factors such as comorbidities and patient preferences (e.g. route of administration, ease of use) $[53,54]$. Like all newer bDMARDs, secukinumab is administered subcutaneously. After appropriate training, secukinumab can be self-administered via a prefilled syringe or pen (Sect. 4). Such devices offer patients convenience and ease of administration, both of which may improve 
their adherence to treatment. In the FUTURE 3 trial, most patients were satisfied or very satisfied with the autoinjector and found it easy or very easy to use (Sect. 2.1.1.3). In realworld clinical practice, secukinumab was associated with high levels of patient and physician satisfaction (Sect. 2.3).

Cost is another factor that can influence the choice of therapy in PsA. Current National Institute for Health and Care Excellence guidance indicates that secukinumab is cost effective in some clinical settings [63]. Semi-Markov models were used to evaluate the cost effectiveness of secukinumab in patients with PsA from the perspectives of the German statutory health insurance system [64] and the Argentinean social security healthcare system [65]. Results suggest that, over a lifetime horizon, secukinumab is cost effective compared with other bDMARDs (adalimumab, certolizumab pegol, etanercept, golimumab, infliximab and ustekinumab) in both biological-experienced patients and biological-naïve patients (without or without concomitant moderate to severe psoriasis) [64, 65]. Additional pharmacoeconomic data relevant to the EU and the USA would be useful.

In conclusion, subcutaneous secukinumab is effective across all key PsA domains and is generally well tolerated, with efficacy and tolerability sustained over the longer term. Thus, secukinumab represents a useful treatment alternative to TNFi and other bDMARDs in adult patients with active PsA.

\section{Data Selection Secukinumab: 692 records identified

$$
\text { Duplicates removed }
$$ \\ Excluded during initial screening (e.g. press releases; news reports; not relevant drug/indication; preclinical study; reviews; case reports; not randomized trial) \\ Excluded during writing (e.g. reviews; duplicate data; small patient number; nonrandomized/phase I/II trials) \\ Cited efficacy/tolerability articles \\ Cited articles not efficacy/tolerability \\ Search Strategy: EMBASE, MEDLINE and PubMed from 2016 to present. Previous Adis Drug Evaluation published in 2016 was hand-searched for relevant data. Clinical trial registries/databases and websites were also searched for relevant data. Key words were secukinumab, Cosentyx, psoriatic arthritis. Records were limited to those in English language. Searches last updated 1 February 2021}

Acknowledgments During the peer review process, the manufacturer of secukinumab was also offered an opportunity to review this article. Changes resulting from comments received were made on the basis of scientific and editorial merit.

\section{Declarations}

Funding The preparation of this review was not supported by any external funding.

Authorship and Conflict of interest H. A. Blair is a salaried employee of Adis International Ltd/Springer Nature, and declares no relevant conflicts of interest. All authors contributed to the review and are responsible for the article content.

Ethics approval, Consent to participate, Consent to publish, Availability of data and material, Code availability Not applicable.

Open Access This article is licensed under a Creative Commons Attribution-NonCommercial 4.0 International License, which permits any non-commercial use, sharing, adaptation, distribution and reproduction in any medium or format, as long as you give appropriate credit to the original author(s) and the source, provide a link to the Creative Commons licence, and indicate if changes were made. The images or other third party material in this article are included in the article's Creative Commons licence, unless indicated otherwise in a credit line to the material. If material is not included in the article's Creative Commons licence and your intended use is not permitted by statutory regulation or exceeds the permitted use, you will need to obtain permission directly from the copyright holder. To view a copy of this licence, visit http://creativecommons.org/licenses/by-nc/4.0/.

\section{References}

1. Hackett S, Coates L. Psoriatic arthritis: an up to date overview. Indian J Rheumatol. 2020;15(1):S45-51.

2. Ogdie A, Coates LC, Gladman DD. Treatment guidelines in psoriatic arthritis. Rheumatology (Oxford). 2020;59(Suppl. 1):i37-46.

3. Toussi A, Maverakis N, Le ST, et al. Updated therapies for the management of psoriatic arthritis. Clin Immunol. 2020. https:// doi.org/10.1016/j.clim.2020.108536.

4. Chimenti MS, D'Antonio A, Conigliaro P, et al. An update for the clinician on biologics for the treatment of psoriatic arthritis. Biologics. 2020;14:53-75.

5. Blauvelt A, Chiricozzi A. The immunologic role of IL-17 in psoriasis and psoriatic arthritis pathogenesis. Clin Rev Allergy Immunol. 2018;55(3):379-90.

6. Novartis. COSENTYX® (secukinumab) injection: US prescribing information. 2020. https://dailymed.nlm.nih.gov. Accessed 18 Jan 2021

7. European Medicines Agency. Secukinumab (Cosentyx): EU summary of product characteristics. 2020. https://www.ema. europa.eu. Accessed 18 Jan 2021.

8. Shirley M, Scott LJ. Secukinumab: a review in psoriatic arthritis. Drugs. 2016;76(11):1135-45.

9. Garnock-Jones KP. Secukinumab: a review in moderate to severe plaque psoriasis. Am J Clin Dermatol. 2015;16(4):323-30.

10. Blair HA. Secukinumab: a review in ankylosing spondylitis. Drugs. 2019;79(4):433-43.

11. Blair HA, Dhillon S. Secukinumab: a review in ankylosing spondylitis. Drugs. 2016;76(10):1023-30.

12. Mease PJ, McInnes IB, Kirkham B. Secukinumab inhibition of interleukin-17A in patients with psoriatic arthritis. N Engl J Med. 2015;373(14):1329-39.

13. McInnes IB, Mease PJ, Kirkham B. Secukinumab, a human antiinterleukin-17A monoclonal antibody, in patients with psoriatic 
arthritis (FUTURE 2): a randomised, double-blind, placebocontrolled, phase 3 trial. Lancet. 2015;386(9999):1137-46.

14. Nash P, Mease PJ, McInnes IB, et al. Efficacy and safety of secukinumab administration by autoinjector in patients with psoriatic arthritis: results from a randomized, placebo-controlled trial (FUTURE 3). Arthritis Res Ther. 2018;20(1):47.

15. Kivitz AJ, Nash P, Tahir H, et al. Efficacy and safety of subcutaneous secukinumab $150 \mathrm{mg}$ with or without loading regimen in psoriatic arthritis: results from the FUTURE 4 study. Rheumatol Ther. 2019;6(3):393-407.

16. Mease P, van der Heijde D, Landewe R, et al. Secukinumab improves active psoriatic arthritis symptoms and inhibits radiographic progression: primary results from the randomised, double-blind, phase III FUTURE 5 study. Ann Rheum Dis. 2018;77(6):890-7.

17. Baraliakos X, Gossec L, Pournara E, et al. Secukinumab in patients with psoriatic arthritis and axial manifestations: results from the double-blind, randomised, phase 3 MAXIMISE trial. Ann Rheum Dis. 2020. https://doi.org/10.1136/annrh eumdis-2020-218808.

18. D'Agostino M, Schett G, Lopez-Rdz A, et al. Secukinumab significantly decreased joint synovitis measured by power doppler ultrasonography in biologic-naive patients with active psoriatic arthritis: primary (12-week) results from a randomized, placebo-controlled phase III study [abstract no. 1361]. In: American College of Rheumatology Convergence. 2020.

19. McInnes IB, Behrens F, Mease PJ, et al. Secukinumab versus adalimumab for treatment of active psoriatic arthritis (EXCEED): a double-blind, parallel-group, randomised, activecontrolled, phase 3b trial. Lancet. 2020;395(10235):1496-505.

20. van der Heijde D, Mease PJ, Landewe RBM, et al. Secukinumab provides sustained low rates of radiographic progression in psoriatic arthritis: 52-week results from a phase 3 study, FUTURE 5. Rheumatology (Oxford). 2020;59(6):1325-34.

21. Mease PJ, Landewe RBM, Rahman P, et al. Subcutaneous secukinumab $300 \mathrm{mg}$ and $150 \mathrm{mg}$ provides sustained inhibition of radiographic progression in psoriatic arthritis over 2 years: results from the phase 3 FUTURE-5 trial [abstract no. LB0006]. Ann Rheum Dis. 2019;78(Suppl. 2):262.

22. McInnes IB, Mease PJ, Ritchlin CT, et al. Secukinumab sustains improvement in signs and symptoms of psoriatic arthritis: 2 year results from the phase 3 FUTURE 2 study. Rheumatology (Oxford). 2017;56(11):1993-2003.

23. Kavanaugh A, Mease PJ, Reimold AM, et al. Secukinumab for long-term treatment of psoriatic arthritis: a two-year followup from a phase III, randomized, double-blind placebo-controlled study. Arthritis Care Res. 2017;69(3):347-55.

24. Mease PJ, Kavanaugh A, Reimold A, et al. Secukinumab provides sustained improvements in the signs and symptoms of psoriatic arthritis: final 5-year results from the phase 3 FUTURE 1 study. ACR Open Rheumatol. 2020;2(1):18-25.

25. Mease PJ, Kavanaugh A, Reimold A, et al. Secukinumab in the treatment of psoriatic arthritis: efficacy and safety results through 3 years from the year 1 extension of the randomised phase III FUTURE 1 trial. RMD Open. 2018. https://doi.org/ 10.1136/rmdopen-2018-000723.

26. Nash P, McInnes IB, Rahman P, et al. Secukinumab provides sustained improvements in the signs and symptoms of active psoriatic arthritis: 3-year results from the phase 3 FUTURE 2 study [abstract no. THU0322]. Ann Rheum Dis. 2018;77(Suppl. 2):379.

27. McInnes IB, Mease PJ, Kivitz AJ, et al. Long-term efficacy and safety of secukinumab in patients with psoriatic arthritis: 5-year (end-of-study) results from the phase 3 FUTURE 2 study. Lancet Rheumatol. 2020;2(4):e227-35.
28. van der Heijde D, Landewe RB, Mease PJ, et al. Secukinumab provides significant and sustained inhibition of joint structural damage in a phase III study of active psoriatic arthritis. Arthritis Rheumatol. 2016;68(8):1914-21.

29. Strand V, Mease P, Gossec L, et al. Secukinumab improves patient-reported outcomes in subjects with active psoriatic arthritis: results from a randomised phase III trial (FUTURE 1). Ann Rheum Dis. 2017;76(1):203-7.

30. McInnes IB, Mease PJ, Schett G, et al. Secukinumab provides rapid and sustained pain relief in psoriatic arthritis over 2 years: results from the FUTURE 2 study. Arthritis Res Ther. 2018;20(1):113.

31. Coates LC, Mease PJ, Gossec L, et al. Minimal disease activity among active psoriatic arthritis patients treated with secukinumab: 2-year results from a multicenter, randomized, double-blind, parallel-group, placebo-controlled phase III study. Arthritis Care Res. 2018;70(10):1529-35.

32. Coates LC, Gladman DD, Nash P, et al. Secukinumab provides sustained PASDAS-defined remission in psoriatic arthritis and improves health-related quality of life in patients achieving remission: 2-year results from the phase III FUTURE 2 study. Arthritis Res Ther. 2018;20(1):272.

33. Coates LC, Nash P, Kvien TK, et al. Comparison of remission and low disease activity states with DAPSA, MDA and VLDA in a clinical trial setting in psoriatic arthritis patients: 2-year results from the FUTURE 2 study. Semin Arthritis Rheum. 2020;50(4):709-18.

34. Chimenti MS, Fonti GL, Conigliaro P, et al. One-year effectiveness, retention rate, and safety of secukinumab in ankylosing spondylitis and psoriatic arthritis: a real-life multicenter study. Expert Opin Biol Ther. 2020;20(7):813-21.

35. Conaghan PG, Holdsworth E, Tian $\mathrm{H}$, et al. Real world effectiveness of secukinumab in psoriatic arthritis: findings from a recent cross sectional survey of rheumatologists and patients in Europe [abstract no. AB0755]. Ann Rheum Dis. 2020;79(Suppl. 1):1674-5.

36. Kiltz U, Peterlik D, Winkelmann V, et al. AQUILA study in Germany - real world data on secukinumab's effectiveness in psoriatic arthritis patients-results from an interim analysis [abstract no. FRI0405]. Ann Rheum Dis. 2019;78(Suppl. 2):889-90.

37. Mease PJ, Blachley T, Glynn M, et al. Secukinumab improves clinical and patient-reported outcomes at 6 months among patients with psoriatic arthritis in the US-based Corrona psoriatic arthritis/ spondyloarthritis (PsA/SpA) registry [abstract no. SAT0429]. Ann Rheum Dis. 2020;79(Suppl. 1):1169.

38. Favalli EG, Marchesoni A, Balduzzi S, et al. Effectiveness and retention rate of secukinumab for psoriatic arthritis and axial spondyloarthritis: real-life data from the Italian LORHEN registry [abstract no. FRI0273]. Ann Rheum Dis. 2020;79(Suppl. 1):722-3.

39. Lorenzin M, Carletto A, Foti R, et al. Effectiveness and safety of secukinumab in naive or TNF-inhibitors failure psoriatic arthritis patients in real life: a 24-months prospective multicenter study [abstract no. FRI0284]. Ann Rheum Dis. 2020;79(Suppl. 1):730.

40. Magrey M, Bozyczko M, Wolin D, et al. Evaluation of the feasibility of a web-based survey to assess patient-reported symptom improvement and treatment satisfaction among patients with psoriatic arthritis receiving secukinumab. Clin Drug Investig. 2019;39(12):1205-12.

41. Martin LM, Valero M, Emperiale V, et al. Real-world experience of secukinumab for psoriatic arthritis [abstract no. FRI0448]. Ann Rheum Dis. 2019;78(Suppl. 2):915.

42. Holdsworth E, Booth N, Lobosco S, et al. Real world physician satisfaction with secukinumab in psoriatic arthritis and ankylosing spondylitis in Europe [abstract no. THU0635]. Ann Rheum Dis. 2019;78(Suppl. 2):611-2.

43. Kiltz U, Peterlik D, Winkelmann V, et al. AQUILA study in Germany - real world adherence and persistence of secukinumab 
treatment in ankylosing spondylitis and psoriatic arthritis patientsan interim analysis [abstract no. AB0705]. Ann Rheum Dis. 2019;78(Suppl. 2):1814-5.

44. Michelsen B, Georgiadis S, Giuseppe DI, D, , et al. Secukinumab effectiveness in 1543 patients with psoriatic arthritis treated in routine clinical practice in 13 European countries in the EuroSpA research collaboration network [abstract no. SAT0430]. Ann Rheum Dis. 2020;79(Suppl. 1):1169-71.

45. Oelke KR, Chambenoit O, Majjhoo AQ, et al. Persistence and adherence of biologics in US patients with psoriatic arthritis: analyses from a claims database. J Comp Eff Res. 2019;8(8):607-22.

46. Letarouilly JG, Flachaire B, Labadie C, et al. Secukinumab and ustekinumab treatment in psoriatic arthritis: results of a direct comparison. Rheumatology (Oxford). 2020. https://doi.org/10.1093/ rheumatology/keaa710.

47. Lindstrom U, Glintborg B, Giuseppe DDI, et al. Comparison of treatment retention of secukinumab and TNF-inhibitors in psoriatic arthritis. Observational data from a Nordic collaboration [abstract no. THU0394]. Ann Rheum Dis. 2020;79(Suppl. 1):431-2.

48. Deodhar A, Mease PJ, McInnes IB, et al. Long-term safety of secukinumab in patients with moderate-to-severe plaque psoriasis, psoriatic arthritis, and ankylosing spondylitis: integrated pooled clinical trial and post-marketing surveillance data. Arthritis Res Ther. 2019;21(1):111.

49. Deodhar A, McInnes I, Baraliakos X, et al. Secukinumab demonstrates a consistent safety profile in patients with psoriasis, psoriatic arthritis and ankylosing spondylitis over long term: updated pooled safety analysis [abstract no. FRI0272]. Ann Rheum Dis. 2020;79(Suppl. 1):722.

50. Elewski BE, Baddley JW, Deodhar AA, et al. Association of secukinumab treatment with tuberculosis reactivation in patients with psoriasis, psoriatic arthritis, or ankylosing spondylitis. JAMA Dermatol. 2020. https://doi.org/10.1001/jamadermatol.2020.3257.

51. Deodhar A, Gladman DD, McInnes IB, et al. Secukinumab immunogenicity over 52 weeks in patients with psoriatic arthritis and ankylosing spondylitis. J Rheumatol. 2020;47(4):539-47.

52. Gossec L, Baraliakos X, Kerschbaumer A, et al. EULAR recommendations for the management of psoriatic arthritis with pharmacological therapies: 2019 update. Ann Rheum Dis. 2020;79(6):700-12.

53. Coates LC, Kavanaugh A, Mease PJ. Group for Research and Assessment of Psoriasis and Psoriatic Arthritis 2015 treatment recommendations for psoriatic arthritis. Arthritis Rheumatol. 2016;68(5):1060-71.

54. Singh JA, Guyatt G, Ogdie A, et al. 2018 American College of Rheumatology/National Psoriasis Foundation guideline for the treatment of psoriatic arthritis. Arthritis Rheumatol. 2019;71(1):5-32.
55. Hetland ML. Psoriatic arthritis: still room for improvement. Lancet. 2020;395(10235):1463-5.

56. McInnes IB, Nash P, Ritchlin C, et al. Secukinumab for psoriatic arthritis: comparative effectiveness versus licensed biologics/apremilast: a network meta-analysis. J Comp Eff Res. 2018;7(11):1107-23.

57. Mourad A, Gniadecki R. Treatment of dactylitis and enthesitis in psoriatic arthritis with biologic agents: a systematic review and metaanalysis. J Rheumatol. 2020;47(1):59-65.

58. Reygaerts T, Mitrovic S, Fautrel B, et al. Effect of biologics on fatigue in psoriatic arthritis: a systematic literature review with meta-analysis. Jt Bone Spine. 2018;85(4):405-10.

59. Simons N, Degboe Y, Barnetche T, et al. Biological DMARD efficacy in psoriatic arthritis: a systematic literature review and metaanalysis on articular, enthesitis, dactylitis, skin and functional outcomes. Clin Exp Rheumatol. 2020;38(3):508-15.

60. Strand V, Husni ME, Betts KA, et al. Network meta-analysis and cost per responder of targeted immunomodulators in the treatment of active psoriatic arthritis. BMC Rheumatology. 2018. https://doi. org/10.1186/s41927-018-0011-1.

61. Strand V, McInnes I, Mease P, et al. Matching-adjusted indirect comparison: secukinumab versus infliximab in biologic-naive patients with psoriatic arthritis. J Comp Eff Res. 2019;8(7):497-510.

62. Kiltz U, Sfikakis PP, Gaffney K, et al. Secukinumab use in patients with moderate to severe psoriasis, psoriatic arthritis and ankylosing spondylitis in real-world setting in Europe: baseline data from SERENA study. Adv Ther. 2020;37(6):2865-83.

63. National Institute for Health and Care Excellence. Certolizumab pegol and secukinumab for treating active psoriatic arthritis after inadequate response to DMARDs. 2017. http://www.nice.org.uk. Accessed 18 Jan 2021.

64. Gandjour A, Ostwald DA. Cost effectiveness of secukinumab versus other biologics and apremilast in the treatment of active psoriatic arthritis in Germany. Appl Health Econ Health Policy. 2020;18(1):109-25.

65. Aiello E, Bianculli PM, Bhattacharyya D, et al. Cost-effectiveness of secukinumab versus other biologics in the treatment of psoriatic arthritis: an Argentinean perspective. Value Health Reg Issues. 2019;20:86-94.

66. Kampylafka E, d'Oliveira I, Linz C, et al. Resolution of synovitis and arrest of catabolic and anabolic bone changes in patients with psoriatic arthritis by IL-17a blockade with secukinumab: results from the prospective PSARTROS study. Arthritis Res Ther 2018;20(1):153. 